\title{
Evaluation des lignées endogames de maïs (Zea mays $L$.) pour le développement des hybrides adaptés aux zones de basse et moyenne altitudes du Cameroun
}

\author{
Arlende Flore NGOMENI ${ }^{1 *}$, Charles $\mathrm{THE}^{2}$, AMOUGOU AKOA $^{3}$ et \\ Vincent de Paul MBOUAPOUOGNIGNI ${ }^{4}$
}

\author{
${ }^{1}$ Programme Céréales, Station IRAD, B.P. 44 Dschang, Cameroun. \\ ${ }^{2}$ West Africa Centre for Crop Improvement, University of Ghana, Legon, School of Agriculture, College of \\ Agriculture and Consumer Sciences, B.P. 30 Legon, Accra, Ghana. \\ ${ }^{3}$ Département de Biologie et Physiologie végétales, Faculté des sciences, Université de Yaoundé 1 , \\ BP 812 Yaoundé, Cameroun. \\ ${ }^{4}$ Programme Légumineuses/Cultures Maraîchères, Station IRAD, B.P. 163 Foumbot, Cameroun. \\ *Auteur correspondant, E-mail : ngflore@yahoo.fr, Tél. : (237)99770469, (237)77256110
}

\section{RESUME}

Un croisement diallèle a été utilisé pour évaluer 11 lignées endogames de maïs (Zea mays L.) de basse et moyenne altitudes dans le développement des hybrides. Les 55 hybrides issus de ce croissement et 3 variétés composites ont été évalués en 2004 sur trois sites dans un dispositif simple comprenant 2 répétitions. Les objectifs étaient d'identifier les hybrides les plus productifs, d'étudier les effets de gènes des lignées et d'évaluer les aptitudes à la combinaison. L'analyse de la variance combinée sur les trois sites a montré que les différents hybrides ont présenté le même comportement et classement relatif d'un site à l'autre pour le rendement. Cinq hybrides à savoir Cam Inb gp 17 x ATP-S4-25W, 91109x ATP-S4-25W, 88094 x cml 254, 88094 x Exp24 et $87036 \mathrm{x} \mathrm{cml} 254$ se sont mieux comportés que le meilleur hybride de l'IRAD (87036 x Exp24). Les lignées de basse altitude ont montré les meilleures performances en croisements dans les deux zones. L'effet de la dominance n'a pas été significatif tout comme l'aptitude spécifique à la combinaison sur les trois sites. Cependant, l'aptitude générale à la combinaison a été significative et en générale positive. Les caractères sont donc contrôlés par les gènes à effets additifs.

(c) 2014 International Formulae Group. All rights reserved.

Mots clés : Aptitude à la combinaison, dominance, effet des gènes.

\section{INTRODUCTION}

Le maïs, céréale la plus cultivée au Cameroun, est consommé par plus du tiers de sa population. Il est la troisième denrée alimentaire produite après le manioc et la banane plantain, et contribue pour plus de 150 milliards de FCFA au produit intérieur brut (MINADER, 2010).

La production camerounaise de maïs s'améliore progressivement au fil des années.
Elle est passée de 738627 tonnes en 2001 à 1674400 tonnes en 2011 (Anonyme, 2012). Toutefois, la production du maïs au Cameroun est toujours déficiente. Le déficit a progressé de 37000 tonnes en 2007 à 120000 tonnes en 2009 (Anonyme, 2012). Pour combler ce déficit, le Cameroun a importé en 2009, 22662 tonnes de maïs à concurrence de 3,870 milliards de FCFA (MINADER, 2010). Cette alternative n'est pas à encourager pour

(C) 2014 International Formulae Group. All rights reserved. DOI : http://dx.doi.org/10.4314/ijbcs.v8i1.23 
un pays en voie de développement dont l'un des défis est d'arriver à nourrir sa population de 19, 6 millions d'habitants qui devrait doubler dans les trente prochaines années.

Le maïs est cultivé dans les 5 zones agro-écologiques du Cameroun, malgré les difficultés pratiques auxquelles sont confrontés les différents acteurs du monde rural à savoir: le manque des semences des variétés améliorées, la non disponibilité des intrants agricoles, la pratique d'une agriculture traditionnelle utilisant peu d'intrants, la fertilité décroissante des sols, les maladies et pestes du maïs, la mauvaise organisation de la filière et du marché.

Au cours des années 80 et au début des années 90, l'unité maïs de l'Institut de Recherche Agronomique (IRA) aujourd'hui Institut de Recherche Agricole pour le Développement (IRAD), avait développé deux pools hétérotiques améliorés pour les zones de moyenne altitude et trois pools hétérotiques pour les zones de basse altitude, à partir desquels des lignées endogames adaptées aux différentes zones agro-écologiques du Cameroun ont été développées. Les croisements entre lignées endogames adaptées aux zones agro-écologiques différentes pourraient permettre une exploitation des facteurs hétérosis. Il existe un intérêt croissant pour le développement des hybrides par les programmes nationaux d'Afrique centrale et de l'ouest (CIMMYT, 1987). Les hybrides de maïs ont l'avantage d'être plus productifs que les meilleures des variétés à pollinisation libre (Moule, 1971). De nombreux auteurs (The et al., 1981; Miranda, 1985 ; Castillo and Goodman, 1989 ; Narro et al., 1996 ; The et al, 1996; Kim, 1997 ; Moll et al., 1998 ; Wende et al., 2007 ; Bassi, 2009 ; Kamtat, 2012) ont travaillé sur l'amélioration génétique du maïs dans les tropiques. Cependant, de tous ces travaux, seuls ceux de The et al. (1996) mettent en exergue les croisements entre parents d'altitude moyenne et de basse altitude. D'où l'intérêt des travaux conduits lors de cette étude.

L'objectif principal de cette étude est de sélectionner des variétés hybrides à haut rendement, adaptées à des zones d'altitudes différentes. Les objectifs spécifiques sont d'identifier les hybrides les plus productifs, d'étudier les effets de gènes des lignées et d'évaluer les aptitudes à la combinaison.

\section{MATERIEL ET METHODES \\ Matériel \\ Matériel génétique}

Le matériel génétique utilisé était constitué de 55 hybrides et de trois composites utilisées comme témoins. Les hybrides ont été issus d'un croisement diallèle entre 3 lignées endogames de maïs adaptées aux altitudes moyennes (AM) et 8 lignées endogames développées pour les basses altitudes (BA). Des trois composites utilisées comme variétés témoins, deux étaient adaptées aux basses altitudes et une aux altitudes moyennes (Tableau 1).

\section{Site expérimental}

Les études ont été menées de mai à septembre 2004 dans trois sites d'expérimentation de l'IRAD.

Le premier site, Njombé (latitude $4^{\circ} 3$ $4^{\circ} 8 \mathrm{~N}$; longitude $9^{\circ} 3-9^{\circ} 8 \mathrm{E} ; 80 \mathrm{~m}$ d'altitude au dessus de la mer) est situé dans la zone de forêt dense humide à pluviométrie monomodale, avec des précipitations moyennes annuelles de $2466 \mathrm{~mm}$ sur sol à cendres volcaniques anciennes. La température moyenne annuelle varie entre $23,6{ }^{\circ} \mathrm{C}$ et $27,3{ }^{\circ} \mathrm{C}$ (CARBAP, 2003).

Le deuxième site, Foumbot (latitude $5^{\circ}$ $5 \mathrm{~N}$; longitude $10^{\circ} 7 \mathrm{E} ; 1100 \mathrm{~m}$ d'altitude au dessus de la mer) se trouve dans la zone des hauts-plateaux de l'Ouest, sur terres noires à cendres volcaniques avec une pluviométrie moyenne annuelle de $1720 \mathrm{~mm}$. La température moyenne annuelle varie entre 16 ${ }^{\circ} \mathrm{C}$ et $27^{\circ} \mathrm{C}$ (Moukam, 1985).

Le troisième site, Ngaoundéré (latitude $7^{\circ}-7^{\circ} 5 \mathrm{~N}$; longitude $13^{\circ} 8$ - $14^{\circ} 3 \mathrm{E} ; 1250 \mathrm{~m}$ d'altitude au dessus de la mer) est situé en zone de haute savane guinéenne avec des précipitations moyennes annuelles de 1300 $\mathrm{mm}$. La température moyenne annuelle varie entre $15^{\circ} \mathrm{C}$ et $30^{\circ} \mathrm{C}$. Le paysage est plat et intercepté de vallées intérieures. Le sol est ferrugineux sur roches basaltiques, riche en sesquioxydes, en fer et en aluminium (MINAGRI, 2000). 
Tableau 1 : Origine institutionnelle génétique, couleur et adaptation présumées des lignées et composites.

\begin{tabular}{lllll}
\hline Variétés & Origine & Adaptation & Couleur & Généalogie \\
\hline Lignées & & & & \\
87036 (a) & IRAD & Moyenne altitude & Blanche & TZMSRx Pop 32 \\
$88094($ a) & IRAD & Moyenne altitude & Blanche & TZMSRx Pop 43 \\
91105 & IRAD & Moyenne altitude & Blanche & TZMSRx Pop 32 \\
M131 & IRAD & Basse altitude & Blanche & TZMSRx Pop 43 \\
Exp 24 & IRAD & Basse altitude & Blanche & TZBSRxPop 43 \\
NCRE gp 28 & IRAD & Basse altitude & Blanche & Pop 43 x TZBSR \\
Cam Inb gp 17 & IRAD & Basse altitude & Jaune & SuwanI - SR \\
ATP-S4-25-W & IRAD & Basse altitude & Blanche & ATP-SR \\
Cml 254 & CIMMYT Mexique & Basse altitude & Blanche & Tuxpeno \\
Cml 365 & CIMMYT Colombie & Basse altitude & Blanche & Pop SA8 \\
Cla 18 & CIMMYT Colombie & Basse altitude & Jaune & Eto \\
& & & & \\
Composites & & & & \\
ATP-SR-Y & IRAD & Basse altitude & Jaune & ATP - SR \\
CMS 8704 & IRAD & Basse altitude & Jaune & SuwanI-SRxPop.28SR \\
Kasaï SR & Zaïre & Moyenne altitude & Blanche & Pop 32x Pop 43
\end{tabular}

Source: Cameroon Inco Annual report year 1999; The et al., 2006. Pop : population ; (a) les lignées 87036 et 88094 sont obtenues sur les BC3 F1 (après 3 rétrocroisements respectivement avec pop32 et pop43); CIMMYT: Centre international pour l'amélioration du maïs et du blé ; IRAD : Institut de Recherche Agricole pour le Développement.

Méthodes

\section{Dispositif expérimental}

Dans chaque site, les génotypes ont été évalués dans un dispositif expérimental en bloc complet randomisé. Le dispositif comportait deux répétitions séparées entre elles par une allée de 1,5 m. L'unité expérimentale était une ligne de $5 \mathrm{~m}$ de longueur comportant 11 poquets distants de 0,5 m l'un de l'autre. La distance entre les lignes était de $0,75 \mathrm{~m}$, deux grains ont été introduits par poquet, avec une densité de semis de 53333 plants à l'hectare. L'essai était entouré de deux lignes de bordure de tous les côtés. La superficie totale de la parcelle était de 663,375 $\mathrm{m}^{2}$.

\section{Mise en place et conduite de l'essai}

Le terrain a été labouré sur tous les sites à l'aide d'une charrue attelée à un tracteur. Les semences ont été comptées et mises dans les enveloppes à raison de 33 graines par enveloppe correspondant à 3 grains par poquet et 11 poquets par ligne.

Le semis a eu lieu à Foumbot et à Ngaoundéré au mois de mai et à Njombé en juin. Les grains ont été enfouis dans les poquets creusés à l'aide d'une machette à une profondeur de 3-4 cm.

Le démariage a été effectué 10 jours après le semis lors du premier épandage d'engrais. Il visait à homogénéiser le nombre de plants par poquet et par ligne. Dans un poquet à trois plants, un plant était supprimé par arrachage manuel. Quand un poquet avait un seul plant, trois plants étaient maintenus si possible dans le poquet suivant dans le but d'atteindre la densité souhaitée de 53333 plants par hectare.

Deux épandages d'engrais minéraux ont eu lieu. Le premier épandage a été effectué 10 jours après le semis avec le NPK (14-5-18 + 4MgO 4S) à la dose de $150 \mathrm{~kg}$ par hectare et l'urée (46-0-0) à la dose de $50 \mathrm{~kg}$ par hectare, ce qui correspond à $22 \mathrm{~N}, 7.5$ $\mathrm{P}_{2} \mathrm{O}_{5}, 27 \mathrm{~K}_{2} \mathrm{O}, 6 \mathrm{MgO}$ et $6 \mathrm{~S}$ unité fertilisante. Le second épandage a été effectué 5 semaines après le semis avec de l'urée (46-0-0) à la dose de $100 \mathrm{~kg}$ par hectare, ce qui correspond à $46 \mathrm{~N}$ unité fertilisante. Au total, l'essai a reçu $(46+27) \mathrm{N}, 7.5 \mathrm{P}_{2} \mathrm{O}_{5}, 27 \mathrm{~K}_{2} \mathrm{O}, 6 \mathrm{MgO}$ et 6 $\mathrm{S}$ unité fertilisante. 
Trois contrôles de mauvaises herbes ont été effectués. Le premier en pré levée après le semis par application du lasso GD (Alachlore $300 \mathrm{~g} / \mathrm{l}+$ Atrazine $180 \mathrm{~g} / \mathrm{l}$ ), à la dose de $150 \mathrm{ml}$ par pulvérisateur de $15 \mathrm{l}$, soit 1.51 de produit par hectare. Le deuxième désherbage a été effectué manuellement quatre semaines après le semis. Le troisième contrôle des mauvaises herbes a été fait dix semaines après le semis par pulvérisation du Gramoxone super à la dose de $150 \mathrm{ml}$ par pulvérisateur de 151 , donc 31 par hectare. Le Gramoxone super a pour matière active le Paraquat dichloride (à $270 \mathrm{~g} / \mathrm{l}$ ), un produit de la famille des dipyridyles. C'est un herbicide de contact.

Pour prévenir l'attaque des chenilles défoliatrices, un insecticide, le Sevin (Carbaryl $850 \mathrm{~g} / \mathrm{kg}$ ) a été pulvérisé à raison d'un sachet par pulvérisateur de 15 litres 6 semaines après le semis, soit 20 sachets par hectare.

La récolte a eu lieu après la maturité physiologique des plants (à 4 mois après le semis). Les épis de chaque unité expérimentale ont été récoltés individuellement à la main, disposés en bout de ligne, comptés, déspathés et pesés.

\section{Mesure des paramètres de production et estimation du rendement}

Le nombre total de plants par ligne a été compté au moment de la récolte. Le poids au champ (PC) des épis récoltés et déspathés sur chaque parcelle expérimentale a été estimé à l'aide d'une balance dont l'unité était le kg.

Le taux d'humidité (TH) a été mesuré après égrainage à l'aide d'un humidimètre à lecture directe.

Le rendement a été estimé pour une humidité totale des graines de $15 \%$. Il a été exprimé en $\mathrm{kg} / \mathrm{ha}$ et s'est calculé à partir de la formule suivante :

$\operatorname{RDT}\left(\mathrm{t}^{-h^{-1}}{ }^{-1}\right)=\frac{\text { PC x } 0,83(100-\mathrm{TH}) \times 10.000 \mathrm{~m}^{2}}{1.000 \times \mathrm{S}}$

$\mathrm{RDT}=$ rendement

$\mathrm{PC}=$ poids au champ de chaque génotype $(\mathrm{kg})$

$\mathrm{TH}=$ taux d'humidité des grains à la récolte

$100-\mathrm{T}=$ différence entre l'humidité maximale et l'humidité des grains à la récolte $\mathrm{S}=$ surface de la parcelle élémentaire
$10.000 \mathrm{~m}^{2}=1$ hectare

$1.000 \mathrm{~kg}=1$ tonne

\section{Analyse statistique}

L'analyse de la variance combinant les sites a été réalisée en utilisant la procédure GLM (General Linear Model) du logiciel SAS. Le modèle statistique utilisé était le suivant :

$Y_{\mathrm{ijr}}=\mu+\mathrm{G}_{\mathrm{i}}+\mathrm{E}_{\mathrm{j}}+(\mathrm{GE})_{\mathrm{ij}}+\mathrm{R}_{\mathrm{j}}+\varepsilon_{\mathrm{ijr}}$

$\mathrm{Y}_{\mathrm{ijr}}=$ observation de chaque variable dans la $\mathrm{r}^{\text {th }}$ répétition, du $\mathrm{ij}^{\text {th }}$ génotype dans le $1^{\text {ème }}$ site

$\mu=$ moyenne générale de la population

$G_{i}$ et $E_{j}$ représentent les effets du $i^{\text {th }}$ génotype et $\mathrm{du} \mathrm{j}^{\text {th }}$ site, quand $(\mathrm{GE})_{\mathrm{ij}}$ représente les interactions génotype - site, $R_{j}$ est l'effet de la répétition du $\mathrm{j}^{\text {th }}$ site, $\varepsilon_{\mathrm{ijr}}$ désigne l'erreur aléatoire obtenue de la $\mathrm{r}^{\text {th }}$ répétition $\mathrm{du} \mathrm{ij}^{\text {th }}$ génotype dans le $\mathrm{j}^{\text {th }}$ site. (ij $=1,2,3, \ldots \ldots \ldots \ldots, 55) \mathrm{j}=1,2,3 ; \mathrm{r}=1,2$.

La séparation des moyennes a été faite par le test de Duncan.

La méthode IV de Griffing (1956) pour laquelle le matériel expérimental comporte uniquement les hybrides F1 (sans réciproques et autofécondations) et, dont le nombre de croisements est obtenu par la formule $\mathrm{n}(\mathrm{n}-1) / 2$, a été appliquée pour la partition de la somme des carrés des croisements en sources de variations dues à l'Aptitude générale à la combinaison (AGC) et à l'Aptitude Spécifique à la Combinaison (ASC). La formule mathématique utilisée pour estimer les effets de l'AGC et ceux de l'ASC se présentait sous le modèle suivant : $\mathrm{Y}_{\mathrm{ijk}}=\mu+\mathrm{r}_{\mathrm{k}}+\mathrm{gi}+\mathrm{g}_{\mathrm{j}}+\mathrm{S}_{\mathrm{ij}}+\mathrm{P}_{\mathrm{ijk}}$

où $\mu=$ moyenne générale

$\mathrm{r}_{\mathrm{k}}=$ effet aléatoire due aux répétitions avec $\mathrm{k}=1,2$

$\mathrm{g}_{\mathrm{i}}$ et $\mathrm{g}_{\mathrm{j}}=$ effets dus à l'aptitude générale à la combinaison (AGC) due au ième et du jème, avec $\mathrm{i}=\mathrm{j}=1 \ldots 11$

$S_{\mathrm{ij}}=$ Effet dû à l'aptitude spécifique à la Combinaison

$\mathrm{P}_{\mathrm{ijk}}=$ erreur aléatoire obtenu à la $\mathrm{k}^{\mathrm{ème}}$ répétition sur le (ij) ${ }^{\text {ème }}$ génotype (Griffing, 1956). 


\section{RESULTATS ET DISCUSSION}

Analyse de la variance combinée sur les trois sites

L'analyse de la variance présentée au Tableau 2 montre que l'interaction site $\mathrm{x}$ accessions n'a pas été significative $(\mathrm{P}>0.05)$ pour le paramètre rendement. Les différents hybrides ont donc présenté le même comportement et classement relatif d'un site à l'autre.

Les accessions, excepté celles évaluées à Foumbot, n'ont pas présenté de différences significatives pour la variable rendement (Tableau 3). Ceci suggère qu'elles ont le même classement relatif d'un site à l'autre.

Il n'y a pas eu de différences significatives entre les sites de l'étude, pourtant ces sites se trouvent dans des zones agro écologiques différentes. La performance moyenne des accessions à Ngaoundéré (4,701 t.ha $\left.{ }^{-1}\right)$ a été meilleure par rapport aux deux autres sites $\left(3,891\right.$ t.ha $^{-1}$ à Njombé et 3,709 t.ha $^{-1}$ à Foumbot) (Tableau 4). La performance des variétés à Njombé a été semblable à celle obtenue à Foumbot, pourtant ces sites ont des altitudes différentes (80 m au-dessus de la mer à Njombé et $1100 \mathrm{~m}$ au-dessus de la mer à Foumbot).

\section{Performance générale des hybrides Rendement moyen en grain sur les trois sites} Le rendement moyen en grain des hybrides testés sur les trois sites a été de 4,1 t.ha ${ }^{-1}$ Les rendements varient de 2,844 t.ha ${ }^{-1}$ pour l'hybride (Cam Inb gp 17 x cml 365) à 6,336 t.ha ${ }^{-1}$ pour l'hybride (Cam Inb gp $17 \mathrm{x}$ ATP-S4-25W). Le composite le plus performant a été ATP-SR-Y avec une production moyenne de 4,637 t.ha ${ }^{-1}$. Parmi les 13 hybrides qui ont été plus performants que le meilleur des composites, 2 ont montré une supériorité de plus de $1 \mathrm{t}$ à l'hectare. Ce sont Cam Inb gp 17 x ATP-S4-25W (6,336 t.ha $\left.{ }^{-1}\right)$ avec $31 \%$ de supériorité et 91105 x ATP-S425W (5,809 t.ha $\left.{ }^{-1}\right)$ avec 25\% de supériorité.

La performance moyenne des 20 meilleurs hybrides présentée au Tableau 5 montre que les rendements varient de 4,319 t.ha $^{-1}$ (M131 x cla 18) à 6,336 t.ha ${ }^{-1}$ (Cam Inb $\mathrm{gp}_{1} 17$ x ATP-S4-25W). Le rendement moyen des hybrides sélectionnés est de 4,922 t.ha ${ }^{-1}$, soit une augmentation de $20 \%$ par rapport à la moyenne de l'essai. Parmi ces meilleurs hybrides, 11 (55\%) proviennent des croisements lignée de basse altitude par lignée d'altitude moyenne avec une performance moyenne de 4,989 t.ha ${ }^{-1}$. Les 9 autres hybrides sont issus des croisements entre les lignées de basse altitude avec une performance moyenne de 4,842 t.ha-1.

Le rendement moyen des croisements d'altitude moyenne (AM) x basse altitude (BA) $\left(4,989\right.$ t.ha $\left.^{-1}\right)$ est semblable à celui des croisements BA x BA (4,842 t.ha $\left.{ }^{-1}\right)$. Aucun croisement AM x AM n'apparaît parmi les 20 meilleurs hybrides. Les deux meilleurs hybrides sur les trois sites sont Cam Inb gp 17 x ATP-S4-25W (6,336 t.ha $\left.{ }^{-1}\right)$ et 91105 x ATPS4-25W $\left(5,809\right.$ t.ha $\left.^{-1}\right)$. Ces deux hybrides sont issus des croisements BA x BA et AM x BA.

La lignée ATP-S4-25W intervient dans 8 des meilleurs croisements, elle est suivie par la lignée 87036 qui apparaît dans 6 croisements. La première lignée est adaptée aux basses altitudes et la deuxième à des altitudes moyennes. Cam Inb gp $\mathrm{g}_{1} 17, \mathrm{cml} 254$ et $\mathrm{NCREgp}_{2} 8$ interviennent chacune dans 4 croisements et ont une adaptation aux basses altitudes. Le meilleur hybride de l'IRAD (87036 x Exp 24) (IRAD, Nkolbisson) a produit en moyenne 5,117 t.ha ${ }^{-1}$. Les 5 hybrides: Cam Inb gp 17 x ATP-S4-25W $\left(6,336\right.$ t.ha $\left.^{-1}\right) ; 91105$ x ATP-S4-25W $(5,809$ t.ha $\left.{ }^{-1}\right) ; 88094$ x cml 254 (5,332 t.ha-1); 88094 x $\operatorname{Exp} 24\left(5,267\right.$ t.ha $\left.^{-1}\right) ; 87036 \mathrm{x} \mathrm{cml} 254$ $\left(5,214\right.$ t.ha $\left.{ }^{-1}\right)$ qui se sont mieux comportés que lui peuvent être retenus comme potentiels hybrides pour une éventuelle vulgarisation.

\section{Rendement en grain en zone d'altitude moyenne}

Dans les zones d'altitude moyenne, la performance des hybrides varie de 2,745 t.ha ${ }^{-1}$ à 5,984 t.ha ${ }^{-1}$. La moyenne de l'essai est de 4,204 t.ha ${ }^{-1}$. La moyenne des 20 meilleurs hybrides est de 5,105 t.ha ${ }^{-1}$. Les meilleurs hybrides proviennent d'un croisement entre une lignée de basse altitude avec une lignée d'altitude moyenne. Ces hybrides ont produit au moins 5,426 t.ha ${ }^{-1}$. La meilleure variété composite est ATP-SR-Y. Elle a produit 4,581 t.ha $^{-1}$. 18 hybrides ont présenté des 
rendements meilleurs que ce composite et 3 hybrides ont été supérieurs d'au moins 1 t. Il s'agit de NCREgp 8 x cla 18 (5,984 t.ha $\left.{ }^{-1}\right)$, 88094 x ATP-S4-25W (5,822 t.ha $\left.{ }^{-1}\right)$ et Cam Inb gp 17 x ATP-S4-25W (5,629 t.ha $\left.{ }^{-1}\right)$.

Parmi les 20 meilleurs hybrides (Tableau 6), 10 (50\%) proviennent des croisements entre une lignée de basse altitude et une lignée d'altitude moyenne avec une performance moyenne de 5,131 t.ha ${ }^{-1}$. Les 10 $(50 \%)$ autres hybrides issus des croisements basse altitude $\mathrm{x}$ basse altitude ont en moyenne produit 5,079 t.ha ${ }^{-1}$. Les hybrides issus des croisements altitude moyenne $x$ altitude moyenne n'ont pas été performants en altitude moyenne. Ce qui est contraire aux résultats de The et al. (1996) qui ont montré dans leur étude que les croisements AM x BA donnent des rendements significativement plus élevés que ceux des croisements AM x AM et BA x BA et aussi, les moyennes en croisement des lignées AM x AM étaient significativement plus élevés que celles des croisements BA x BA. De plus, 50\% des meilleurs hybrides sont issus des croisements $\mathrm{AM} \times \mathrm{BA}$, ce qui suggère leur appartenance à des pools hétérotiques différents. L'absence des croisements AM x AM parmi les 20 meilleurs suggérerait que les lignées d'altitude moyenne de notre étude appartiendraient au même pool hétérotique et de ce fait, montrent très peu d'hétérosis.

Les résultats obtenus sur les deux sites d'altitude moyenne montrent que les meilleurs hybrides ont au moins un parent de basse altitude dans leur généalogie. Les lignées NCRE gp $_{2} 8$ et Cam Inb gp 17 interviennent chacune dans 6 croisements. Ces deux lignées sont adaptées aux basses altitudes. Elles sont suivies des lignées ATP-S4-25W et 87036 qui interviennent dans 5 croisements chacune, puis de M131 et cla 18 qui apparaissent dans 4 croisements. Ce qui suggère que ces lignées de basse altitude contiennent des gènes d'adaptation aux altitudes moyennes, et peuvent donc être utilisées dans la création des hybrides adaptés aux altitudes moyennes si elles ne sont pas sensibles aux maladies foliaires du maïs qui prévalent dans ces zones (H ; Turcicum et Puccicum ...).

\section{Rendement moyen en grain sur le site de Foumbot}

Les rendements sur le site de Foumbot varient de 1,762 t.ha $^{-1}$ pour l'hybride NCREgp 28 x ATP-S4-25W à 5,769 t.ha ${ }^{-1}$ pour Cam Inb $\operatorname{grp}_{1} 17$ x ATP-S4-25W. La moyenne de l'essai est de 3,708 t.ha ${ }^{-1}$. Le meilleur témoin composite ATP-SR-Y, a produit 4,068 t.ha ${ }^{-1}$. Les rendements de 9 hybrides ont été supérieurs à celui de ce composite d'au moins 1 t.ha ${ }^{-1}$.

Les rendements varient de 4,178 t.ha $^{-1}$ (Cam Inb gp 17 x cla 18) à 5,769 t.ha ${ }^{-1}(\mathrm{Cam}$ Inb $\mathrm{gp}_{1} 17 \mathrm{x}$ ATP-S4-25W) pour les 20 meilleurs hybrides, soit une différence de 1,591 t.ha ${ }^{-1}$ (Tableau 7). Tous ces hybrides ont donné des rendements supérieurs à celui du composite Kasaï SR. La supériorité de ces hybrides par rapport au composite varie de $174 \%$ à $359 \%$.

A Foumbot, la moyenne des croisements AM $\mathrm{x}$ BA $\left(4,883 \mathrm{t}^{-h^{-1}}{ }^{-1}\right)$ est semblable à celle des croisements BA x BA $\left(4,971 \mathrm{t} \cdot \mathrm{ha}^{-1}\right)$. Aucun croisement AM x AM ne figure parmi les 20 meilleurs. Les deux meilleurs hybrides sont Cam Inb $\mathrm{gp}_{1} 17 \mathrm{x}$ ATP-S4-25W (5,769 t.ha $\left.{ }^{-1}\right)$ et 91105 x Cam Inb $\mathrm{gp}_{1} 17\left(5,769\right.$ t.ha $\left.{ }^{-1}\right)$. Ces deux hybrides sont issus des croisements BA x BA et AM x BA. Parmi les 20 meilleurs hybrides, Cam Inb $\mathrm{gp}_{1} 17$ et $\mathrm{cml} 254$, lignées de basse altitude, interviennent respectivement dans 7 et 6 croisements comme le parent 87036, lignée d'altitude moyenne qui intervient dans 6 croisements.

\section{Rendement moyen en grain sur le site de Ngaoundéré}

Les rendements des hybrides sur le site de Ngaoundéré varient de 2,111 t.ha ${ }^{-1}$ pour l'hybride ATP-S4-25W x cla 18 à 7,966 t.ha ${ }^{-1}$ pour l'hybride M131 x ATP-S4-25W. La moyenne de l'essai est de 4,700 t.ha ${ }^{-1}$. Le meilleur témoin composite ATP-SR-Y, a produit 5,093 t.ha ${ }^{-1}$. Les rendements de 10 hybrides ont été supérieurs à celui de ce composite d'au moins 1 t.ha $^{-1}$.

Les rendements des 20 meilleurs hybrides varient de 5,307 t.ha ${ }^{-1}(87036 \mathrm{x}$ NCRE $\left.\mathrm{gp}_{2} 8\right)$ à 7,966 t.ha-1 (M131 x ATP-S4$25 \mathrm{~W})$, soit une différence de 2,659 t.ha $^{-1}$ 
(Tableau 8). Tous ces hybrides ont donné des rendements supérieurs à celui de Kasaï SR. La supériorité de ces hybrides par rapport au composite varie de $12 \%$ à $68 \%$.

A Ngaoundéré, la moyenne des croisements AM x BA $\left(6,125\right.$ t.ha $\left.^{-1}\right)$ est semblable à celle des croisements BA x BA $\left(6,137\right.$ t.ha $\left.^{-1}\right)$ et supérieure à celle des croisements AM x AM $\left(5,418\right.$ t.ha $\left.^{-1}\right)$. Les deux meilleurs hybrides sont M131 x ATPS4-25W (7,966 t.ha $\left.{ }^{-1}\right)$ et 88094 x Exp24 $\left(7,110\right.$ t.ha $\left.^{-1}\right)$. Ces deux hybrides sont issus des croisements AM x BA.

A Ngaoundéré, $\mathrm{NCREgp}_{2} 8$ qui est une lignée adaptée aux basses altitudes apparaît 6 fois comme parent parmi les 20 meilleurs. Cette lignée est suivie par M131, ATP-S4-25W et cla 18 qui apparaissent 5 fois chacune et les lignées 87036, 88094 et Cam Inb gp $_{1} 17$ qui sont utilisées 4 fois chacune. Des 7 lignées citées plus haut, 2 sont d'altitude moyenne et 5 de basse altitude. Ceci suggère une fois de plus que les lignées de basse altitude peuvent servir au développement des hybrides pour les altitudes moyennes.

En comparant les rendements en grain de Foumbot à ceux de Ngaoundéré, on se rend compte que les rendements sont plus élevés à Ngaoundéré d'au moins 1 t.ha ${ }^{-1}$. Enfin, seuls 6 hybrides ont été parmi les meilleurs à Foumbot et à Ngaoundéré.

\section{Rendement moyen en grain sur le site de Njombé}

Les rendements des hybrides sur le site de Njombé varient de 1,293 t.ha ${ }^{-1}$ pour l'hybride Exp24 x Cam Inb gp 17 à 8,434 t.ha ${ }^{-1}$ pour l'hybride $88094 \mathrm{x} \mathrm{cml} \mathrm{254.} \mathrm{Le}$ meilleur témoin composite ATP-SR-Y, a produit 4,750 t.ha ${ }^{-1}$. Les rendements de 7 hybrides ont été supérieurs à celui de ce composite d'au moins 1 t.ha ${ }^{-1}$.

Les rendements sont de l'ordre de 4,138 t.ha ${ }^{-1}\left(91105 \mathrm{x} \mathrm{cml} \mathrm{365)} \mathrm{à} \mathrm{8,434} \mathrm{t.ha}{ }^{-1}\right.$ (88091 x cml 254) pour les 20 meilleurs hybrides, soit une différence de 4,296 t.ha ${ }^{-1}$ (Tableau 9). Le rendement moyen de l'hybride est de 4,922 t.ha ${ }^{-1}$. Les 11 hybrides qui ont donné des rendements supérieurs à celui de ATP-SR-Y sont : 88094 x cml 254 $\left(8,434\right.$ t.ha $\left.{ }^{-1}\right)$; Cam Inb gp 17 x ATP-S4-25-W $\left(7,751\right.$ t.ha $\left.^{-1}\right) ; \quad$ Exp 24 x ATP-S4-25-W $\left(7,591\right.$ t.ha $\left.{ }^{-1}\right) ; 87036 \times \operatorname{Exp} 24$ (7,332 t.ha $\left.{ }^{-1}\right)$; 91105 x Cam Inb gp 17 (7,146 t.ha $\left.{ }^{-1}\right) ; \mathrm{cml}$ $254 \times$ cml 365 (6,323 t.ha $\left.{ }^{-1}\right)$; Cam Inb gp 17 $\mathrm{x}$ cla $18\left(6,105\right.$ t.ha $\left.^{-1}\right) ; 87036 \mathrm{x} \mathrm{cml} 254$ $\left(5,684\right.$ t.ha $\left.{ }^{-1}\right)$; ATP-S4-25-W x cml 365 $\left(5,313 \quad\right.$ t.ha $\left.{ }^{-1}\right) ; 88094$ x $\operatorname{Exp} 24(5,310$ t.ha $\left.^{-1}\right)$; 91105 x ATP-S4-25-W (5,200 t.ha $\left.{ }^{-1}\right)$. La supériorité de ces hybrides par rapport au composite varie de $9 \%$ à $76 \%$.

A Njombé, la moyenne des croisements BA x BA (5,647 t.ha $\left.{ }^{-1}\right)$ est semblable à celle des croisements BA x AM (5,692 t.ha $\left.{ }^{-1}\right)$ et supérieure à celle des croisements $\mathrm{AM}$ x AM $\left(4,267\right.$ t.ha $\left.^{-1}\right)$. Les deux meilleurs hybrides sont 88094 x cml $254\left(8,434\right.$ t.ha $\left.^{-1}\right)$ et Cam Inb $\mathrm{gp}_{1} 17$ x ATP-S4-25-W (7,751 t.ha $\left.{ }^{-1}\right)$. Ces deux hybrides sont issus des croisements AM $\mathrm{x}$ BA et BA $x$ BA.

A Njombé, ATP-S4-25W qui est une lignée de basse altitude, apparaît 7 fois comme parent parmi les 20 meilleurs hybrides. Cette lignée est suivie par $\mathrm{cml} 254$ et 91105 qui apparaissent 5 fois chacune et 88094, Cam Inb gp 17 , cml 365 qui sont utilisées 4 fois chacune. Des lignées suscitées, 4 sont adaptées aux basses altitudes.

\section{Effet des gènes}

La dominance ne joue pas un très grand rôle, car les génotypes ont presque le même hétérosis (Tableau 10). Par conséquent, les hybrides peuvent être recombinés entre eux pour fabriquer une population synthétique.

\section{Aptitudes à la combinaison \\ Aptitude spécifique à la combinaison}

L'aptitude spécifique à la combinaison (ASC) n'a pas été significative sur les trois sites pour la variable rendement (Tableau 11), ce qui sous entend qu'aucun hybride ne peut servir de base de classification des lignées en pools hétérotiques. 


\section{Aptitude générale à la combinaison}

L'aptitude générale à la combinaison (AGC) est significative sur les trois sites (Tableau 11). Elle est en général, positive (Tableau 12). Ceci suggère que les caractères sont contrôlés par les gènes à effets additifs. L'amélioration des populations peut donc se faire par une simple sélection récurrente. Par contre, les ASC et AGC ont montré des effets significatifs ainsi qu'une prédominance des effets additifs des gènes dans les travaux de Thé et al. (1996).

A Njombé, Foumbot et Ngaoundéré, 6, 4 et 6 parents ont présenté une AGC positive (Tableau 12). Les meilleurs géniteurs dans ces sites sont respectivement ATP-S4-25-W, cml 254, Cam Inb gp $17,88094,87036$ et 91105 ; Cam Inb gp 17,87036 , cml 254 et ATP-S425W ; NCREgp 28, M131, ATP-S4-25-W, 87036, 88094 et Exp24. Les lignées M131 et 88094 ont également eu des AGC positives en zone d'altitude moyenne sur sol à forte fertilisation azotée (Kamtat, 2012), tout comme les lignées ATP-S4-25-W, M131,
Cam Inb $\mathrm{gp}_{1} 17$ et 87036 en zone d'altitude moyenne sur sol acide (Thé et al., 2006). L'apparition de 3 lignées de basse altitude parmi les 4 lignées de Foumbot confirme une fois de plus que les lignées de basse altitude peuvent servir de donneurs de gènes lors du développement des hybrides pour les altitudes moyennes.

Seulement 2 lignées présentent aux deux sites d'altitude moyenne des AGC positives : ce sont ATP-S4-25W et 87036.

Les deux meilleurs parents à Njombé (ATP-S4-25W et cml 254), à Foumbot (Cam Inb $\mathrm{gp}_{1} 17$ et 87036$)$ et à Ngaoundéré (NCREgp 8 et M131) peuvent servir de géniteurs pour leurs sites respectifs dans un programme d'amélioration, mais en général, quelle que soit l'altitude, deux lignées ont présenté des AGC positives dans les trois sites. Il s'agit des parents 87036 et ATP-S425W. Il ressort également des travaux de plusieurs auteurs (The et al., 1996 ; Thé et al., 2006 ; Kamtat, 2012) que la lignée 87036 a eu une AGC positive.

Tableau 2: Carré moyen issu de l'analyse de la variance pour la variable rendement en grain sur les trois sites.

\begin{tabular}{lcc}
\hline Source de variation & Ddl & Rendement en grain \\
\hline Répétition & 1 & $3.99^{\mathrm{NS}}$ \\
Site & 2 & $31.76^{* * *}$ \\
Accessions & 56 & $5.59^{\mathrm{NS}}$ \\
Site $\mathrm{x}$ Accessions & 112 & $3.72^{\mathrm{NS}}$ \\
Erreur & 112 & 3.46 \\
\hline Valeur de $\mathrm{F}: 0.16 ;$ Pr $>\mathrm{F}: 0.1692$ & & \\
$* * *:$ hautement significatif à $\mathrm{p}<0.001$ & ** : significatif à $\mathrm{p}<0.01$ \\
$*:$ significatif à $\mathrm{p}<0.05 \quad \mathrm{NS}:$ non significatif & Ddl : degré de liberté
\end{tabular}

Tableau 3 : Carré moyen issu de l'analyse de la variance par site pour la variable rendement.

\begin{tabular}{|c|c|c|c|c|}
\hline Source de variation & Ddl & Njombé & Foumbot & Ngaoundéré \\
\hline Répétition & 1 & $81,75 * * *$ & $11,2 * *$ & $4,99^{\mathrm{NS}}$ \\
\hline Accessions & 56 & $5,24^{\mathrm{NS}}$ & $2,44 *$ & $3,35^{\mathrm{NS}}$ \\
\hline Erreur & 56 & 4,34 & 1,34 & 3,14 \\
\hline
\end{tabular}


Tableau 4 : Rendement moyen en grain par site.

\begin{tabular}{lc}
\hline Site & Rendement en grain \\
\hline Njombé & $3.891 \mathrm{a}$ \\
Foumbot & $3.709 \mathrm{a}$ \\
Ngaoundéré & $4.701 \mathrm{~b}$ \\
\hline Moyenne & 4.100 \\
C.V & 45.380 \\
P.P.D.S & 0.487 \\
\hline \multicolumn{2}{l}{ Les lettres a et b permettent de séparer les moyennes } \\
C.V = coefficient de variation \\
P.P.D.S = plus petite différence significative \\
NS : non significatif \\
Ddl : degré de liberté
\end{tabular}

Tableau 5 : Les 20 meilleurs hybrides sur les trois sites.

\begin{tabular}{|c|c|c|c|}
\hline Hybrides & & Rendement & Adaptation \\
\hline Cam Inb gp 17 x ATP-S4-25W & & $6.336 \mathrm{a}$ & $\mathrm{BA} \times \mathrm{BA}$ \\
\hline 91105 x ATP-S4-25W & & $5.809 \mathrm{ab}$ & $\mathrm{AM} \times \mathrm{BA}$ \\
\hline 88094 x cml 254 & & $5.332 \mathrm{abc}$ & $\mathrm{AM} \times \mathrm{BA}$ \\
\hline 88094 x Exp 24 & & $5.267 \mathrm{abc}$ & $\mathrm{AM} \times \mathrm{BA}$ \\
\hline $87036 \times \mathrm{cml} 254$ & & $5.214 \mathrm{abc}$ & $\mathrm{AM} \times \mathrm{BA}$ \\
\hline 87036 x Exp 24 & & $5.117 \mathrm{abcd}$ & $\mathrm{AM} \times \mathrm{BA}$ \\
\hline Exp 24 x ATP-S4-25W & & 5.051 abcde & $\mathrm{BA} \times \mathrm{BA}$ \\
\hline $\mathrm{Cml} 254 \times \mathrm{cml} 365$ & & 5.047 abcde & $\mathrm{BA} \times \mathrm{BA}$ \\
\hline 88094 x ATP-S4-25W & & 5.047 abcde & $\mathrm{AM} \times \mathrm{BA}$ \\
\hline $87036 \times$ ATP-S4-25W & & 4.936 abcdef & $\mathrm{AM} \times \mathrm{BA}$ \\
\hline $91105 \times$ x ATP-S4-25W & & 4.927 abcdef & $\mathrm{AM} \times \mathrm{BA}$ \\
\hline NCRE $\mathrm{gp}_{2} 8 \times$ cla 18 & & 4.925 abcdef & $\mathrm{BA} \times \mathrm{BA}$ \\
\hline M131 x Cam Inb gp 17 & & 4.809 abcdef & $\mathrm{AM} \times \mathrm{BA}$ \\
\hline 87036 x cla 18 & & 4.512 abcdef & $\mathrm{AM} \times \mathrm{BA}$ \\
\hline NCRE $\mathrm{gp}_{2} 8 \times$ ATP-S4-25W & & 4.400 abcdef & $\mathrm{BA} \times \mathrm{BA}$ \\
\hline $87036 \times$ NCRE $\mathrm{gp}_{2} 8$ & & 4.393 abcdef & $\mathrm{AM} \times \mathrm{BA}$ \\
\hline ATP-S4-25W x cml 254 & & 4.370 abcdef & $\mathrm{BA} \times \mathrm{BA}$ \\
\hline NCRE $\mathrm{gp}_{2} 8 \times$ Cam Inb gp 17 & & 4.320 abcdef & $\mathrm{BA} \times \mathrm{BA}$ \\
\hline 87036 x Cam Inb gp 17 & & 4.320 abcdef & $\mathrm{AM} \times \mathrm{BA}$ \\
\hline M131 x cla 18 & & 4.319 abcdef & $\mathrm{AM} \times \mathrm{BA}$ \\
\hline ATP-SR-Y & & 4.637 abcdef & BA \\
\hline Kasaï SR & & $3.026 \mathrm{def}$ & $\mathrm{AM}$ \\
\hline Moyenne des sélectionnés & 4.922 & & \\
\hline Moyenne de l'essai & 4.100 & & \\
\hline P.P.D.S & 2.121 & & \\
\hline C.V & 45.38 & & \\
\hline S.E & 3.46 & & \\
\hline Moyenne BA x BA (45\%) & 4.842 & & \\
\hline Moyenne BA x AM (55\%) & 4.989 & & \\
\hline
\end{tabular}


Tableau 6 : Les 20 meilleurs hybrides en fonction du rendement en zone d'altitude moyenne.

\begin{tabular}{|c|c|c|c|c|}
\hline Hybrides & & Entrée & Rendement & Adaptation \\
\hline NCRE gp $_{2} 8 \times$ cla18 & & 45 & $5.984 \mathrm{a}$ & $\mathrm{BA} \times \mathrm{BA}$ \\
\hline 88094 x ATP-S4-25W & & 31 & $5.822 \mathrm{ab}$ & $\mathrm{AM} \times \mathrm{BA}$ \\
\hline Cam Inb gp 17 x ATP-S4-25W & & 46 & $5.629 \mathrm{abc}$ & $\mathrm{BA} \times \mathrm{BA}$ \\
\hline 87036 x NCRE $\mathrm{gp}_{2} 8$ & & 5 & $5.426 \mathrm{abcd}$ & $\mathrm{AM} \times \mathrm{BA}$ \\
\hline Exp $24 \times$ NCRE $\mathrm{gp}_{2} 8$ & & 35 & 5.313 abcde & $\mathrm{BA} \times \mathrm{BA}$ \\
\hline 87036 x cla18 & & 10 & 5.307 abcde & $\mathrm{AM} \times \mathrm{BA}$ \\
\hline 88094 x Exp 24 & & 28 & 5.245 abcdef & $\mathrm{AM} \times \mathrm{BA}$ \\
\hline 91105 x Cam Inb gp 17 & & 15 & 5.140 abcdefg & $\mathrm{AM} \times \mathrm{BA}$ \\
\hline NCRE $\mathrm{gp}_{2} 8$ x Cam Inb $\mathrm{gp}_{1} 17$ & & 41 & 5.081 abcdefg & $\mathrm{BA} \times \mathrm{BA}$ \\
\hline 87036 x ATP-S4-25W & & 7 & 5.033 abcdefgh & $\mathrm{AM} \times \mathrm{BA}$ \\
\hline 87036 x cml 254 & & 8 & 4.979 abcdefghi & $\mathrm{AM} \times \mathrm{BA}$ \\
\hline M131 x ATP-S4-25W & & 24 & 4.977 abcdefghi & $\mathrm{AM} \times \mathrm{BA}$ \\
\hline M131 x Cam Inb gp 17 & & 23 & 4.951 abcdefghij & $\mathrm{AM} \times \mathrm{BA}$ \\
\hline 88094 x Cam Inb gp 17 & & 30 & 4.950 abcdefghij & $\mathrm{AM} \times \mathrm{BA}$ \\
\hline M131 x NCRE $\mathrm{gp}_{2} 8$ & & 22 & 4.919 abcdefghij & $\mathrm{AM} \times \mathrm{BA}$ \\
\hline Exp 24 x cla 18 & & 40 & 4.912 abcdefghij & $\mathrm{BA} \times \mathrm{BA}$ \\
\hline 91105 x ATP-S4-25W & & 16 & 4.791 abcdefghijk & $\mathrm{AM} \times \mathrm{BA}$ \\
\hline 87036 x Cam Inb gp 17 & & 6 & 4.624 abcdefghijk & $\mathrm{AM} \times \mathrm{BA}$ \\
\hline NCRE $\operatorname{gp}_{2} 8 \times \mathrm{cml} 365$ & & 44 & 4.518 abcdefghijk & $\mathrm{BA} \times \mathrm{BA}$ \\
\hline M131 x cla18 x & & 27 & 4.512 abcdefghijk & $\mathrm{AM} \times \mathrm{BA}$ \\
\hline ATP- SR-Y & & 56 & 4.581 abcdefghijk & BA \\
\hline Kasaï SR & & 58 & 3.137 ghijk & $\mathrm{AM}$ \\
\hline Moyenne des sélectionnés & 5.105 & & & \\
\hline Moyenne de l'essai & 4.204 & & & \\
\hline P.P.D.S & 2.092 & & & \\
\hline C.V & 35.524 & & & \\
\hline S.E & 2.231 & & & \\
\hline Moyenne BA x BA (50\%) & 5.070 & & & \\
\hline Moyenne BA x AM (50\%) & 5.131 & & & \\
\hline
\end{tabular}

Tableau 7 : Les 20 meilleurs hybrides en Fonction du rendement à Foumbot.

\begin{tabular}{|c|c|c|}
\hline Hybrides & Rendement & Adaptation \\
\hline Cam Inb gp 17 x ATP-S4-25W & $5.769 \mathrm{a}$ & $\mathrm{AB} \times \mathrm{AB}$ \\
\hline 91105 x Cam Inb gp 17 & $5.769 \mathrm{a}$ & $\mathrm{AM} \times \mathrm{AB}$ \\
\hline 87036 x Cam Inb gp 17 & $5.657 \mathrm{ab}$ & $\mathrm{AM} \times \mathrm{AB}$ \\
\hline $87036 \times$ NCRE $\mathrm{gp}_{2} 8$ & $5.544 \mathrm{abc}$ & $\mathrm{AM} \times \mathrm{AB}$ \\
\hline M131 x cml 254 & $5.430 \mathrm{abcd}$ & $\mathrm{AM} \times \mathrm{AB}$ \\
\hline 87036 x ATP-S4-25W & 5.427 abcd & $\mathrm{AM} \times \mathrm{AB}$ \\
\hline Exp24 x NCRE $\mathrm{gp}_{2} 8$ & $5.422 \mathrm{abcd}$ & $\mathrm{AB} \times \mathrm{AB}$ \\
\hline NCRE $\operatorname{gp}_{2} 8 \times$ cla1 8 & 5.193 abcde & $\mathrm{AB} \times \mathrm{AB}$ \\
\hline Cam Inb gp 17 x cml254 & 5.193 abcde & $\mathrm{AB} \times \mathrm{AB}$ \\
\hline 87036 x cml 254 & 4.980 abcdef & $\mathrm{AM} \times \mathrm{AB}$ \\
\hline 88094 x ATP-S4-25W & 4.980 abcdef & $\mathrm{AM} \times \mathrm{AB}$ \\
\hline ATP-S4-25W x cml 254 & 4.748 abcdefg & $\mathrm{AB} \times \mathrm{AB}$ \\
\hline 87036 x cla 18 & 4.628 abcdefgh & $\mathrm{AM} \times \mathrm{AB}$ \\
\hline
\end{tabular}




\begin{tabular}{|c|c|c|c|}
\hline Exp24 x Cam Inb gp 17 & & 4.412 abcdefghi & $\mathrm{AB} \times \mathrm{AB}$ \\
\hline NCRE $\mathrm{gp}_{2} 8 \times$ Cam Inb $\mathrm{gp}_{1} 17$ & & 4.393 abcdefghi & $\mathrm{AB} \times \mathrm{AB}$ \\
\hline 87036 x Exp24 Exp24 & & 4.184 abcdefghij & $\mathrm{AM} \times \mathrm{AB}$ \\
\hline 88094 x cml 254 & & 4.183 abcdefghij & $\mathrm{AM} \times \mathrm{AB}$ \\
\hline 88094 x Cam Inb gp 17 & & 4.183 abcdefghij & $\mathrm{AM} \times \mathrm{AB}$ \\
\hline 99105 x cml 254 & & 4.182 abcdefghij & $\mathrm{AM} \times \mathrm{AB}$ \\
\hline Cam Inb gp 17 x cla 18 & & 4.178 abcdefghij & $\mathrm{AB} \times \mathrm{AB}$ \\
\hline ATP-SR-Y & & 4.068 abcdefghijk & BA \\
\hline Kasaï SR & & 1.2561 & AM \\
\hline Moyenne des sélectionnés & 4.922 & & \\
\hline Moyenne de l'essai & 3.708 & & \\
\hline P.P.D.S & 2.324 & & \\
\hline C.V & 31.285 & & \\
\hline SE & 1.346 & & \\
\hline Moyenne BA x BA (45\%) & 4.971 & & \\
\hline Moyenne BA x AM (55\%) & 4.883 & & \\
\hline
\end{tabular}

Tableau 8 : Les 20 meilleurs hybrides en fonction du rendement à Ngaoundéré.

\begin{tabular}{|c|c|c|c|}
\hline Hybrides & & Rendement & Adaptation \\
\hline M131 x ATP-S4-25W & & $7.966 \mathrm{a}$ & $\mathrm{AM} \times \mathrm{BA}$ \\
\hline 88094 x Exp24 & & $7.110 \mathrm{ab}$ & $\mathrm{AM} \times \mathrm{BA}$ \\
\hline NCRE $\operatorname{gp}_{2} 8 \times$ cla1 8 & & $6.776 \mathrm{abc}$ & $\mathrm{BA} \times \mathrm{BA}$ \\
\hline 88094 x ATP-S4-25W & & $6.665 \mathrm{abc}$ & $\mathrm{AM} \times \mathrm{BA}$ \\
\hline Exp24 x cla18 & & $6.329 \mathrm{abcd}$ & $\mathrm{BA} \times \mathrm{BA}$ \\
\hline 91105 x ATP-S4-25W & & 6.199 abcde & $\mathrm{AM} \times \mathrm{BA}$ \\
\hline M131 x Cam Inb gp 17 & & 6.178 abcde & $\mathrm{AM} \times \mathrm{BA}$ \\
\hline NCRE $\mathrm{gp}_{2} 8 \times \mathrm{cml} 365$ & & 6.128 abcde & $\mathrm{BA} \times \mathrm{BA}$ \\
\hline $91105 \times \operatorname{Exp} 24$ & & 6.105 abcde & $\mathrm{AM} \times \mathrm{BA}$ \\
\hline $91105 x$ cla 18 & & 6.103 abcde & $\mathrm{AM} \times \mathrm{BA}$ \\
\hline $87036 \times$ cla 18 & & 5.986 abcdef & $\mathrm{AM} \times \mathrm{BA}$ \\
\hline 87036 x M131 & & 5.936 abcdef & $\mathrm{AM} \times \mathrm{AM}$ \\
\hline NCRE $\mathrm{gp}_{2} 8 \times$ Cam Inb $\mathrm{gp}_{1} 17$ & & 5.770 abcdefg & $\mathrm{BA} \times \mathrm{BA}$ \\
\hline M131 x NCRE $\mathrm{gp}_{2} 8$ & & 5.769 abcdefg & $\mathrm{AM} \times \mathrm{BA}$ \\
\hline 88094 x Cam Inb gp 17 & & 5.716 abcdefg & $\mathrm{AM} \times \mathrm{BA}$ \\
\hline M131 x cla18 & & 5.647 abcdefgh & $\mathrm{AM} \times \mathrm{BA}$ \\
\hline Cam Inb gp ${ }_{1} 17$ x ATP-S4-25W & & 5.488 abcdefgh & $\mathrm{BA} \times \mathrm{BA}$ \\
\hline $87036 \times 88094$ & & 5.418 abcdefgh & $\mathrm{AM} \times \mathrm{AM}$ \\
\hline NCRE $\mathrm{gp}_{2} 8 \times$ ATP-S4-25W & & 5.317 abcdefgh & $\mathrm{BA} \times \mathrm{BA}$ \\
\hline 87036 x NCRE $\operatorname{gp}_{2} 8$ & & 5.307 abcdefgh & $\mathrm{AM} \times \mathrm{BA}$ \\
\hline ATP- SR- Y & & 5.093 abcdefgh & BA \\
\hline Kasaï SR & & 4.748 abcdefgh & $\mathrm{AM}$ \\
\hline Moyenne des sélectionnés & 6.095 & & \\
\hline Moyenne de l'essai & 4.700 & & \\
\hline P.P.D.S & 3.552 & & \\
\hline C.V & 37.725 & & \\
\hline S.E & 3.144 & & \\
\hline Moyenne BA x BA (50\%) & 6.137 & & \\
\hline Moyenne BA x AM (45\%) & 6.125 & & \\
\hline Moyenne AM x AM (5\%) & 5.418 & & \\
\hline
\end{tabular}


Tableau 9 : Les 20 meilleurs hybrides en fonction du rendement à Njombé.

\begin{tabular}{|c|c|c|c|}
\hline Hybrides & & Rendement & Adaptation \\
\hline $88094 \times \mathrm{cml} 254$ & & $8.434 \mathrm{a}$ & $\mathrm{AM} \times \mathrm{BA}$ \\
\hline Cam Inb gp 17 x ATP-S4-25-W & & $7.751 \mathrm{ab}$ & $\mathrm{BA} \times \mathrm{BA}$ \\
\hline Exp 24 x ATP-S4-25-W & & $7.591 \mathrm{abc}$ & $\mathrm{BA} \times \mathrm{BA}$ \\
\hline 87036 x Exp 24 & & $7.332 \mathrm{abcd}$ & $\mathrm{AM} \times \mathrm{BA}$ \\
\hline 91105 x Cam Inb gp 17 & & 7.146 abcde & $\mathrm{AM} \times \mathrm{BA}$ \\
\hline $\mathrm{cml} 254 \mathrm{x} \mathrm{cml} 365$ & & 6.323 abcdef & $\mathrm{BA} \times \mathrm{BA}$ \\
\hline Cam Inb gp 17 x cla 18 & & 6.105 abcdefg & $\mathrm{BA} \times \mathrm{BA}$ \\
\hline 87036 x cml 254 & & 5.684 abcdefgh & $\mathrm{AM} \times \mathrm{BA}$ \\
\hline ATP-S4-25-W x cml 365 & & 5.313 abcdefghi & $\mathrm{BA} \times \mathrm{BA}$ \\
\hline 88094 x Exp 24 & & 5.310 abcdefghi & $\mathrm{AM} \times \mathrm{BA}$ \\
\hline 91105 x ATP-S4-25-W & & 5.200 abcdefghi & $\mathrm{AM} \times \mathrm{BA}$ \\
\hline ATP-S4-25-W x cml 254 & & 4.751 abcdefghi & $\mathrm{BA} \times \mathrm{BA}$ \\
\hline 87036 x ATP-S4-25-W & & 4.743 abcdefghi & $\mathrm{AM} \times \mathrm{BA}$ \\
\hline 88094 x cla 18 & & 4.638 abcdefghi & $\mathrm{AM} \times \mathrm{BA}$ \\
\hline M131 x Cam Inb gp 17 & & 4.523 abcdefghi & $\mathrm{AM} \times \mathrm{BA}$ \\
\hline NCRE gp $_{2} 8 \times$ ATP-S4-25-W & & 4.300 abcdefghi & $\mathrm{BA} \times \mathrm{BA}$ \\
\hline 91105 x cml 254 & & 4.298 abcdefghi & $\mathrm{AM} \times \mathrm{BA}$ \\
\hline 91105 x 88094 & & 4.267 abcdefghi & $\mathrm{AM} \times \mathrm{AM}$ \\
\hline NCRE $\mathrm{gp}_{2} 8 \times \mathrm{cml} 365$ & & 4.165 bcdefghi & BA x BA \\
\hline 91105 x cml 365 & & 4.138 bcdefghi & $\mathrm{AM} \times \mathrm{BA}$ \\
\hline ATP-SR- Y & & 4.750 abcdefghi & BA \\
\hline Kasaï SR & & 2.805 fghi & AM \\
\hline Moyenne des selectionnés & 5.600 & & \\
\hline Moyenne de l'essai & 3.890 & & \\
\hline P.P.D.S & 4.175 & & \\
\hline C.V & 53.571 & & \\
\hline SE & 4.344 & & \\
\hline Moyenne BA x BA (45\%) & 5.647 & & \\
\hline Moyenne BA x AM (50\%) & 5.692 & & \\
\hline Moyenne AM x AM (5\%) & 4.267 & & \\
\hline
\end{tabular}

Tableau 10 : Valeur moyenne en croisement des groupes d'accessions par zone.

\begin{tabular}{|c|c|c|c|c|c|c|c|c|}
\hline \multirow[t]{3}{*}{ Génotype } & \multicolumn{6}{|c|}{ Altitude moyenne } & \multirow{2}{*}{\multicolumn{2}{|c|}{$\begin{array}{c}\text { Basse altitude } \\
\text { Njombé }\end{array}$}} \\
\hline & \multicolumn{2}{|r|}{ Foumbot } & \multicolumn{2}{|c|}{ Ngaoundéré } & \multicolumn{2}{|r|}{ Moyenne } & & \\
\hline & $\%$ & RDT (t.ha' ${ }^{-1}$ ) & $\%$ & RDT (t.ha' $\left.{ }^{-1}\right)$ & $\%$ & RDT (t.ha $\left.{ }^{-1}\right)$ & $\%$ & RDT(t.ha $\left.{ }^{-1}\right)$ \\
\hline $\mathrm{BA} \times \mathrm{BA}$ & 40 & 4.91 & 30 & 5.97 & 30 & 5.24 & 40 & 5.78 \\
\hline BA $x$ AM & 60 & 4.93 & 60 & 5.68 & 70 & 5.05 & 55 & 4.26 \\
\hline $\mathrm{AM} \times \mathrm{AM}$ & - & - & 10 & 6.22 & - & - & 5 & 5.58 \\
\hline
\end{tabular}

BA : basse altitude; AM : altitude moyenne; RDT: rendement en grain \%: pourcentage d'apparition des groupes d'accessions 
Tableau 11 : Carré moyen issu de l'analyse de la variance des aptitudes à la combinaison.

\begin{tabular}{|c|c|c|c|c|}
\hline Source de variation & Ddl & Njombé & Foumbot & Ngaoundéré \\
\hline Hybrides & 54 & $5.4^{\mathrm{NS}}$ & $2.34 *$ & 3.5 \\
\hline AGC & 10 & $8.4^{*}$ & $4.53 * *$ & $4.5^{*}$ \\
\hline ASC & 44 & $4.7^{\mathrm{NS}}$ & $1.84^{\mathrm{NS}}$ & $3.2^{\mathrm{NS}}$ \\
\hline Erreur & 54 & 4.34 & 1.34 & 3.14 \\
\hline
\end{tabular}

Tableau 12: Aptitude générale à la combinaison des parents sur les trois sites.

\begin{tabular}{lccc}
\hline Parents & Njombé & Foumbot & Ngaoundéré \\
\hline 87036 & 0.115 & 0.621 & 0.237 \\
88094 & 0.223 & -0.368 & 0.097 \\
M131 & -1.092 & -0.548 & 0.669 \\
91105 & 0.057 & -0.177 & -0.003 \\
Exp 24 & -0.055 & -0.166 & 0.430 \\
NCREgp $_{2} 8$ & -1.029 & -0.020 & 0.737 \\
Cam Inb gp 17 & 0.265 & 1.014 & -0.330 \\
ATP-S4-25-W & 1.220 & 0.020 & 0.254 \\
Cml 254 & 0.782 & 0.420 & -0.797 \\
Cml 365 & -0.179 & -0.676 & -0.803 \\
Cla 18 & -0.305 & -0.118 & -0.103 \\
\hline
\end{tabular}

\section{Conclusion}

Les hybrides de notre étude ont présenté le même hétérosis et classement relatifs d'un site à l'autre pour le rendement en grain. Ils peuvent donc être testés sur un site. Les cinq hybrides à savoir Cam Inb gp 17 x ATP-S4-25W (6,336 t.ha $\left.{ }^{-1}\right), 91105$ x ATPS4-25W (5,809 t.ha $\left.{ }^{-1}\right), 88094$ x cml 254(5,332 t.ha $\left.{ }^{-1}\right), 88094 \times \operatorname{Exp} 24\left(5,267\right.$ t.ha $\left.^{-1}\right)$ et $87036 \times$ cml $254\left(5,214\right.$ t.ha $\left.^{-1}\right)$ qui se sont mieux comportés que le meilleur hybride de l'IRAD (87036 x Exp24) qui a produit en moyenne 5,117 t.ha $^{-1}$ devraient être multipliés et vulgarisés auprès des producteurs. Les lignées de basse altitude peuvent servir de donneur de gènes lors du développement des hybrides pour les altitudes moyennes.

La dominance n'a pas joué un grand rôle dans cette étude. Les accessions peuvent être recombinées entre elles pour fabriquer une population synthétique.

Aucun hybride ne peut servir de base de classification des lignées en pools hétérotiques. Les caractères sont contrôlés par les gènes à effets additifs. L'amélioration des populations issues de la recombinaison de ces lignées peut donc se faire par une simple sélection récurrente.

Les lignées de basse altitude de cette étude devraient être testées dans différentes zones d'altitude moyenne pour observer leur sensibilité aux maladies foliaires qui prévalent dans ces écologies. Les lignées d'altitude moyenne appartenant au même pool hétérotique devraient être recombinées entre elles pour l'obtention des variétés synthétiques.

\section{REMERCIEMENTS}

Le matériel végétal utilisé pour cette étude a été mis à notre disposition par l'Institut de Recherche Agricole pour le Développement (IRAD) de Nkolbisson à Yaoundé. Les auteurs remercient particulièrement cet institut et étendent leur gratitude à M. Célicard GUEPI ZONKENG pour son assistance technique pendant l'analyse des données. 


\section{REFERENCES}

Anonyme 2012. Statistiques. FAO.

Bassi O. 2009. Contribution des top-cross et hybrides variétaux à l'amélioration du maïs (Zea mays L.) sur sol pauvre en azote. Mémoire de DESS. Université de Yaoundé I, Yaoundé, 35 p.

CARBAP. 2003. Rapport annuel des données climatologiques. CARBAP.

Castillo-Gonzalez F, Goodman MM. 1989. Agronomic evaluation of Latin American maize accessions. Crop Sci., 29: 853-861.

CYMMIT 1987. Results of diallel trial. A technical report presented at the USCIMMYT Combining Ability Workshop. Ed Batun, Mexico 27-30 May 1987, CIMMYT, MEXICO D.E.

Grifting B. 1956. Concept of general and specific combining ability in relation to diallel crossing systems. J. Biol. Sci., 9: 463-493.

Kamtat FG. 2012. Evaluation des hybrides simples d'un diallèle de maïs (Zea mays L.) issus de lignées efficientes à l'utilisation de l'azote dans le sol. Mémoire de MPIS. Université de Yaoundé 1, Yaoundé, 47 p.

Kim SK. 1997. Achievements, challenges and future direction of hybrid maize research and production in West and Central Africa. In: Badu-Apraku B, Akoroda MO, Ouedraogo M, Quin FM (Eds.), Proc. of a Regional Maize Workshop, June, 1995, IITA, Cotonou, Benin Republic, Pp. 4282.

MINADER. 2010. Comice agropastoral Ebolowa. MINADER, Cameroun.

MINAGRI. 2000. Caractérisation des principales terres agricoles du Cameroun. FASA (UDs), Dschang, 124 p.

Miranda FJB. 1985. Breeding methodologies for tropical maize. In Breeding Strategies for Maize Production Improvement in the Tropic Firenze, Brandolini A, Salamine F (eds). FAO, Instituti Agronomico per l'Oltremare.
Moll RH, Salbuana WS, Robinson HF. 1998. Heterosis and Genetic diversity in variety crosses in maize: Crop Sci., 28: 752 758.

Moukam L. 1985. Etude des Sols et des Contraintes du Verger de la Station IRA de Foumbot. IRA : Foumbot.

Moule C. 1971. Céréales, Phytotechnique Spéciale. La Maison Rustique. Masson : Paris ; $235 \mathrm{p}$.

Narro L, Pandey S, Crossa J, De Leonc. Salazarf. 2003. Using Line $\mathrm{x}$ Tester interaction for the formation of yellow maize synthetics tolerant to acid soils. Crop Sci., 43: 1718-1728.

The C, Ayuk-Takem JA, Mongmong B. 1981. Heterotic Groups in Low-Land Tropical Maize. Agronomy Abstract. American Society of Agronomy: Madison, Wisconsin; 118.

The C, Zonkeng C, Enyong L. 1996. Heterosis of low-land $\mathrm{x}$ mid-altitude crosses of Tropical maize. In strategy for sustainable maize production in Wewt Central Africa. Proceedings of a Regional Maize Workshop 21-25 April 1997. IITA Cotonou, Benin Republic.

The C, Calba H, Zonkeng C, Ngonkeu MEL, Adetimirin VO, Mafouasson HA, Meka SS, Horst WJ. 2006. Responses of maize grain yield to changes in acids soils characteristics after soil amendments. Plant and Soil, 284: 45-57.

Thé C, Mafouassom H, Mbouemboue P, Zonkeng C, Tagne A, Horst WJ. 2006. Identification de groupes hétérotiques pour la tolérance du maïs (Zea mays L.) aux sols acides des tropiques. Cahiers Agricultures, 15(4): 337 - 346.

Wende A, Mosisa W, Birhanu T, Legesse W, Diallo A, Twumasi A. 2007. Performances of CYMMIT maize germplasm under low - N soil conditions in the mid-altitude sub humid agroecology of Ethiopia. African Crop Science Conference Proceedings, 8: 1518. 